\title{
Abstracts from Nippon Eiseigaku Zasshi (Japanese Journal of Hygiene) vol. 66 no. 1
}

(C) The Japanese Society for Hygiene 2011

\section{"Molecular-Targeting Prevention" of Cancer - The Theory and its Possibilities}

Nippon Eiseigaku Zasshi, 66, 3-12 (2011)

Toshiyuki SAKAI

Department of Molecular-Targeting Cancer Prevention, Kyoto Prefectural University of Medicine

In previous prevention studies, molecular targets were not intended. We then proposed the concept termed "molecular-targeting prevention" and applied it to cancer prevention. In most malignant tumors, tumor-suppressor genes, the retinoblastoma gene (RB) and/or the p53 gene are considered to be inactivated. We therefore hypothesized that $\mathrm{RB}$ and/or p53 might be good candidates for the molecular-targeting prevention of cancer. Interestingly, many cancer-preventive food factors were found to reactivate the lost functions of RB and/or p53 by a "gene-regulating chemoprevention" strategy. We next proposed the concept termed "combination-oriented molecular-targeting prevention", in which only the preventive effects are synergistically enhanced. We then investigated the TNF-related apoptosis-inducing ligand (TRAIL)-death receptor 5 (DR5) pathway as a candidate of the target, and found that many cancer-preventive food factors could enhance the pathway resulting in the synergistic apoptosis of various cancer cells. We hope that these strategies will contribute to the prevention of cancer.

\section{Epidemiologic Review of Long-Term, Low-Level Exposure to Environmental Chemicals and Cardiovascular Disease: An Exposure-Response Relationship}

Nippon Eiseigaku Zasshi, 66, 13-21 (2011)

Toru TAKEBAYASHI

Department of Preventive Medicine and Public Health, Keio University School of Medicine

The effects of exposure to environmental chemicals, such as heavy metals and fine particulates, on cardiovascular diseases have been reported. To set a permissible exposure standard, an exposureresponse relationship should be elucidated. Thus, epidemiologic evidence in relation to long-term, low-level exposure to environmental chemicals (i.e., lead, cadmium, mercury, arsenic, $\mathrm{PM}_{2.5}$, carbon disulfide) was reviewed. As a result, there exists a clear exposure-response relationship between exposure to lead or $\mathrm{PM}_{2.5}$ and cardiovascular diseases, but those cohort studies were carried out in the US only. From epidemiologic viewpoint, "consistency" of the observed relationship must be clarified, especially findings of a study in the Japanese population. It is well known that the distribution pattern of cardiovascular risk factors are quite different between two countries, which could modify the true relationship. This will require studies of large samples from the general Japanese population with control for potential confouders. Moreover, to detect a small effect of exposure within low-level range, random misclassification of exposure as well as that of effect must be minimized. Thus, for studies of heavy metals, sensitive biomarkers identified in toxicological studies would be applied. For $\mathrm{PM}_{2.5}$, a wide range of population samples should be covered by a national sampling network in the near future. Finally, the integration of epidemiologic and toxicological evidence would be more important for risk assessment of environmental chemicals at low exposure levels, because an exposure-response relationship from only epidemiologic observation may be unstable with a wide confidence interval around the threshold.

\section{Physician Shortage in Japan: The New Postgraduate Medical Education Program and Physicians as a Human Medical Resource}

Nippon Eiseigaku Zasshi, 66, 22-28 (2011)

Kyoko NOMURA

Department of Hygiene and Public Health,

Teikyo University School of Medicine

Japan now faces a serious physician shortage. After introducing the new postgraduate medical education (PGME) system and doctor-tofacility matching system, residents shifted their teaching hospitals from university hospitals to non-university hospitals. Because university hospitals had played a central role in allocating physicians to communities, the decrease in the number of physicians at university hospitals has driven this physician shortage. Japanese policymakers blame the new PGME for exacerbating this physician shortage and have tentatively agreed to reform the PGME to encourage residents to 
return to university hospitals. However, the PGME system should not be reformed only for political reasons; such a change requires a scientific basis. First, after the introduction of the new PGME, residents showed an improved clinical competence; therefore, it has accomplished its ultimate goal. Second, the residents' satisfaction level in terms of the residency system and clinical skills training was significantly higher at non-university hospitals than at university hospitals. This implies that training conditions at university hospitals are not as good as at non-university hospitals, which explains the decrease in the number of physicians at university hospitals. Third, in 2009, the Japanese government increased the maximum medical school enrollment to mitigate the physician shortage. However, a simple increase does not solve the problem of physician shortage unless it also addresses the problem of physician maldistribution. Fourth, the number of females entering medicine is increasing, and women constituted $30 \%$ of newly certified physicians in 2010. In this era of physician shortage, female physicians are highly recommended as a human medical resource.

\section{Importance of Twin Research in Preventive Medicine and Future Perspective -Gene-Environment Interaction Research in Epigenetics Era-}

\author{
Nippon Eiseigaku Zasshi, 66, 29-30 (2011) \\ Kazuo HAYAKAWA \\ Center for Twin Research, Graduate School of Medicine, \\ Osaka University
}

The importance of twin research in the field of preventive medicine is described from the viewpoint of gene-environment interaction. The recent advancements in twin research in Japan and other countries are the major topics in this paper. The historical background of the Japan Society for Twin Studies is described. The Center for Twin Research of Osaka University is also described as the first center of this kind in Japan. The advancement of epigenetic research is described as a new global trend of twin research, particularly in European countries. Other new trends in twin research in Asian countries, such as China, Indonesia, Russia, Iran, and Malaysia, are also described.

\section{Life Course Genetic Epidemiologic Study Based on Longitudinal Twin-Family Data: A New Perspective}

\author{
Nippon Eiseigaku Zasshi, 66, 31-38 (2011) \\ Syuichi OOKI \\ Department of Health Science, Ishikawa Prefectural \\ Nursing University
}

Family-based life course epidemiology is the approach to clarify longterm effects of accumulations and chains of biological and social risk exposures in one's life trajectory, namely, before and during conception, infancy, childhood, young adulthood and later adult life. Both genetic and environmental factors are considered. One of the theoretical rationales is the 'developmental origin of health and disease (DOHaD)' hypothesis. According to this theory, the causes of adult diseases are attributed to predictive adaptive responses and biological programming or epigenetic changes during a critical period of early life. If the environment later in life after birth is different from what is predicted, a mismatch has happened, leading to the development of a disease. This theory is supported by many epidemiologic studies, animal experiments, and evolution theory of biology. Longitudinal twin-family data are very powerful and useful information in the family-based life course genetic epidemiologic study.

\section{Perspective for Molecular Epidemiologic Cohort Study of Lifestyle-Related Disease}

Nippon Eiseigaku Zasshi, 66, 39-41 (2011)

Hirohisa IMAI $^{1}$ and Kuninori SHIWAKU ${ }^{2}$

${ }^{1}$ Department of Epidemiology, National Institute of Public Health

${ }^{2}$ Department of Environmental and Preventive Medicine,

Shimane University School of Medicine

The 80th annual meeting of the Japanese Society of Hygiene was held this year in Sendai. The authors hosted a symposium entitled "Perspective for molecular epidemiologic cohort study of lifestyle-related disease," at which four guests served as panelists. Through the courtesy of Editor-in-Chief Dr. Ohtsuki, space in the current issue has been devoted to a "mini feature" on the symposium to provide briefings by two panelists, Dr. Kurokawa and Dr. Hata. Here, the authors provide an introduction to the briefings, describe the current status of research and state their expectations for the future.

\section{Association of BMI with the Beta3 Adrenergic Receptor Gene Mutation: A Meta-Analysis}

Nippon Eiseigaku Zasshi, 66, 42-46 (2011)

Naoyuki KUROKAWA

Environmental Health Sciences, Graduate school of medicine, Tohoku University

The beta3 adrenergic receptor (ADRB3) is a part of the adrenergic system, which is known to play a key role in energy metabolism. The relationship between the Trp64Arg variant of ADRB3 and body mass index (BMI) has been widely examined; however, the results of these studies have been inconsistent. Therefore, we performed a metaanalysis of the relationship between an ADRB3 variant and BMI in 2008. Our results suggested that the Trp64Arg variant of ADRB3 was associated with BMI in East Asians, but not Europeans. Additionally, our report showed the importance of meta-analyses in the field of genetic association studies for common traits. In 1995, Yoshida et al. reported that the Trp64Arg variant of ADRB3 was related to difficulty in weight loss. The decrease in body weight in obese subjects with the mutation was lower than that in obese subjects without the mutation following a 3 month combined low-calorie diet and exercise regimen. However, subsequent studies have yielded inconsistent results. Accordingly, further studies are needed to reliably assess and interpret gene-phenotype associations with this ADRB3 variant.

\section{Genome-Wide Association Study for Ischemic Stroke Based on the Hisayama Study}

Nippon Eiseigaku Zasshi, 66, 47-52 (2011)

Jun HATA ${ }^{1}$, Michiaki KUBO ${ }^{2}$ and Yutaka KIYOHARA ${ }^{1}$

${ }^{1}$ Department of Environmental Medicine, Graduate School

of Medical Sciences, Kyushu University

${ }^{2}$ Laboratory for Genotyping Development, Center for Genomic Medicine, RIKEN

Ischemic stroke is a major cause of death and disability, and occurs owing to a combination of multiple environmental and genetic risk factors. Although twin studies and family-based studies have suggested the existence of genetic risk factors for ischemic stroke, few candidate genes have been discovered. To identify genes for susceptibility to ischemic stroke, we performed a genome-wide 
association study using 52,608 single nucleotide polymorphism (SNP) markers. After comparison of allele frequencies between 1,112 patients with ischemic stroke and the same number of age- and sexmatched control subjects, who were selected from among the residents of the town of Hisayama, Japan, we identified three novel candidate genes for ischemic stroke: $P R K C H$ [protein kinase $\mathrm{C}$ eta (PKC $\eta$ )], AGTRL1 (apelin receptor), and ARHGEF10 (Rho guanine nucleotide exchange factor 10). In the functional analyses, we found that $\mathrm{PKC} \eta$ was expressed mainly in vascular endothelial cells and foamy macrophages in human atherosclerotic lesions, and that the kinase activity of PKC $\eta$ was modified by a nonsynonymous SNP in $P R K C H$ (rs2230500). We also clarified that functional SNPs in AGTRL1 (rs9943582) and ARHGEF10 (rs4376531) affected transcriptional activities owing to the different Sp1-binding affinities. In a 14-year follow-up cohort study of Hisayama residents, all of these SNPs were significantly associated with the development of ischemic stroke. Although the mechanisms of these genes in the pathogenesis of ischemic stroke are still to be elucidated, our findings might contribute to a better understanding of ischemic stroke in the future.

\section{Application of Electrogastrography to Public Health}

Nippon Eiseigaku Zasshi, 66, 54-63 (2011)

Yasuyuki MATSUURA $^{1}$, Tetsuya YAMAMOTO ${ }^{2,3}$, Masumi TAKADA ${ }^{3,4}$, Tomoki SHIOZAWA ${ }^{5}$ and Hiroki TAKADA ${ }^{3,6}$

${ }^{1}$ Department of Information Engineering, Graduate School of Information Science, Nagoya University

${ }^{2}$ Department of Radiology, Gifu University of Medical Science

${ }^{3}$ The Second Department of Physiology, Aichi Medical University School of Medicine

${ }^{4}$ Department of Occupational and Environmental Health, Nagoya City University Graduate School of Medical Sciences ${ }^{5}$ Department of Business Administration, School of Business, Aoyama Gakuin University

${ }^{6}$ Department of Human and Artificial Intelligent Systems, Graduate School of Engineering, University of Fukui

In general, gastrointestinal motility tests cause pain; therefore, the establishment of noninvasive methods is desired. Noninvasive methods facilitate the measurement of motility close to the normal physiological state, can provide new findings, and may contribute to the development of associated fields. Electrogastrography (EGG) is a gastrointestinal motility test in which gastrointestinal electric activity is measured. Compared with other gastrointestinal motility measurement methods such as the gastric emptying and internal pressure measurement methods, EGG is noninvasive and allows measurement under minimum restriction; therefore, measurement for a long time is also possible. In addition, since gastrointestinal electric activity, which cannot be quantified using other methods, can be measured, EGG is applicable to the evaluation of the state of the body and pathological conditions, and may provide new findings such as those useful for the prevention of gastrointestinal dysfunction associated with various disorders. EGG is also useful for preventing disorders associated with abnormal gastrointestinal activity such as functional dyspepsia, which has been more frequently observed in recent years, and constipation, which is an extremely frequent complaint in the elderly. Thus, EGG is of marked importance in public health. However, the range of EGG utilizations and applications is still limited at present. Therefore, we outlined the measurement/analysis methods, the advantages and problems of EGG and electrogastroenterography (EGEG), described their clinical importance, and also commented on forefront studies on EGG and evaluated its prospects.

\section{Analysis of Electrogastrography in Gastrectomized Subjects}

Nippon Eiseigaku Zasshi, 66, 64-70 (2011)

Masumi TAKADA $^{1,2}$, Tetsuya YAMAMOTO ${ }^{1,3}$, Hiroki TAKADA ${ }^{1,4}$, Kiyoko YOKOYAMA ${ }^{5}$, Masaru MIYAO ${ }^{6}$ and Yasuyuki MATSUURA ${ }^{6}$

${ }^{1}$ The Second Department of Physiology, Aichi Medical University School of Medicine

${ }^{2}$ Department of Occupational and Environmental Health, Nagoya City University Graduate School of Medical Sciences

${ }^{3}$ Department of Radiology, Gifu University of Medical Science

${ }^{4}$ Department of Human and Artificial Intelligent Systems,

Graduate School of Engineering, University of Fukui

${ }^{5}$ Graduate School of Design and Architecture,

Nagoya City University

${ }^{6}$ Department of Information Engineering, Graduate School

of Information Science, Nagoya University

Objectives: To establish a method for the development of a mathematical model of autonomic activity in gastrointestinal movements and to basically evaluate of the application of the nonlinear analysis method to electrogastrography, we performed feature extraction of electrogastrographic changes in healthy elderly and gastrectomized subjects.

Methods: The subjects consisted of 9 healthy elderly males and 3 elderly males without constipation who had undergone resection of $2 / 3$ of the stomach. Electrogastrograms were obtained in a sitting position for $30 \mathrm{~min}$ and in a supine position for $90 \mathrm{~min}$. Spectrum analyses of electrographic time series, the maximum Lyapunov exponent for the evaluation of the chaos of dynamic systems forming time series, and translation error for the evaluation of the smoothness of the attractor orbit were performed.

Results: The maximum Lyapunov exponent was a positive number in all analysis intervals in all subjects. This suggests the irregularity of electrogastrograms in gastrectomized subjects. The translation error in the gastrectomized subjects was higher than that in the healthy elderly subjects, showing irregularity. However, as a result of spectrum analysis, gastric electric activity was predominant on electrogastrograms of the healthy elderly subjects, but intestinal electric activity was predominant in the gastrectomized subjects.

Conclusions: Differentiation between healthy and gastrectomized elderly people is difficult using only one of the spectrum analysis methods, the maximum Lyapunov exponent, or the translation error. However, since the extracted features differed among the 3 analysis methods, differentiation and diagnosis may be possible using a combination of these methods.

\section{Electrogastrography in the Elderly and Effect of Hot Stupes on the Solar Plexus}

Nippon Eiseigaku Zasshi, 66, 71-76 (2011)

Tetsuya YAMAMOTO ${ }^{1,2}$, Yasuyuki MATSUURA ${ }^{3}$, Hiroki

TAKADA $^{4}$, Satoshi IWASE ${ }^{1}$ and Junichi SUGENOYA ${ }^{1}$ 
${ }^{1}$ Second Department of Physiology, Aichi Medical University

${ }^{2}$ Department of Radiological Technology,

Gifu University of Medical Science

${ }^{3}$ Department of Information Engineering, Graduate School of Information Science, Nagoya University

${ }^{4}$ Department of Human and Artificial Intelligent Systems,

Graduate School of Engineering, University of Fukui

Objectives: Constipation affects as many as $26 \%$ of elderly men and $34 \%$ of elderly women and is a problem that has been related to a diminished quality of life. The aim of this study was to investigate the influence of aging on electrogastrograms (EGGs). On the basis of the EGGs of elderly individuals, the effect of hot stupe application on the epigastrium was also evaluated.

Methods: The first group included 14 healthy, young participants (age 20-25 years) and the second group comprised 14 healthy, elderly participants (age 65-76 years). EGGs were obtained for the first $30 \mathrm{~min}$ in a sitting position and for the next period $(>150 \mathrm{~min})$ in a supine position. A hot stupe was placed on the epigastrium of each elderly patient 90 min after the postural change. We evaluated EGGs by spectral analysis and nonlinear analysis.

Results: On comparing the gastrointestinal electrical activity of the elderly group with that of the young group, the activity in the former revealed an overall decrease. With hot stupe application, translation error in the elderly individuals was significantly larger than that without hot stupe application $(p<0.01)$. We also observed a gender-specific characteristic, wherein the gastrointestinal motility of the young women was likely only influenced by postural change. Moreover, the translation error in the EGGs of the elderly individuals increased during hot stupe heating.

Conclusions: This increase in the translation error suggests that the electrical synergy between the stomach and intestine could be enhanced by the application of a hot stupe. Our findings also indicated that hot stupe application on the epigastrium was effective for improving gastrointestinal motility in elderly individuals.

\section{Fungal Biota in Manned Space Environment and Impact on Human Health}

Nippon Eiseigaku Zasshi, 66, 77-82 (2011)

Koichi MAKIMURA $^{1,2}$, Kazuo SATOH ${ }^{2}$, Takashi SUGITA ${ }^{3}$ and Takashi YAMAZAKI ${ }^{4}$

${ }^{1}$ Laboratory of Space and Environmental Medicine,

Graduate School of Medicine, Teikyo University

${ }^{2}$ Institute of Medical Mycology and Genome Research Center, Teikyo University

${ }^{3}$ Department of Microbiology, Meiji Pharmaceutical University

${ }^{4}$ Japan Aerospace and Exploration Agency

It is important to promote microbiological research essential for long-term manned space activities under microgravity and in a completely closed environment in space craft in relation to longduration space expeditions on the International Space Station (ISS) or to the moon and Mars in the future. Environmental monitoring data from the space shuttle, the Mir, and the ISS have already shown that microorganisms isolated from air and on inner surfaces of space craft were generally carried by crew members. The Japanese Experiment Module (JEM) "KIBO" was attached to the ISS and started its operation from 2008. It is an invaluable opportunity to begin the survey of the transition of microbiota, particularly fungal biota, in JEM from "brand-new" to "well-used" condition at various periods. Therefore, we are preparing the on-board analyzing systems for microbiota in air and on inner surfaces of ISS/JEM and normal microbiota of the astronauts themselves. In this paper, we introduce the current status and future plans on fungal research on ISS/JEM to protect flight crew members and flight hardware from potentially hazardous microorganisms from the environmental and biomedical aspects of Japan Aerospace Exploration Agency (JAXA).

\section{Reconstituting Evaluation Methods Based on both Qualitative and Quantitative Paradigms}

Nippon Eiseigaku Zasshi, 66, 83-94 (2011)

Hiroaki MIYATA ${ }^{1}$, Suguru OKUBO ${ }^{1}$, Satoru YOSHIE ${ }^{2}$

and Ichiro $\mathrm{KAI}^{3}$

${ }^{1}$ Department of Healthcare Quality Assessment, Graduate School of Medicine, The University of Tokyo

${ }^{2}$ Center for Biomedical Ethics and Law, The University of Tokyo ${ }^{3}$ Department of Social Gerontology, Graduate School of Medicine, The University of Tokyo

Debate about the relationship between quantitative and qualitative paradigms is often muddled and confusing and the clutter of terms and arguments has resulted in the concepts becoming obscure and unrecognizable. In this study we conducted content analysis regarding evaluation methods of qualitative healthcare research. We extracted descriptions on four types of evaluation paradigm (validity/credibility, reliability/credibility, objectivity/confirmability, and generalizability/transferability), and classified them into subcategories. In quantitative research, there has been many evaluation methods based on qualitative paradigms, and vice versa. Thus, it might not be useful to consider evaluation methods of qualitative paradigm are isolated from those of quantitative methods. Choosing practical evaluation methods based on the situation and prior conditions of each study is an important approach for researchers.

\section{Concentrations of Persistent Organochlorine Pesticides in Whole Blood of Pregnant Women in Hokkaido Study on Environment and Children's Health}

Nippon Eiseigaku Zasshi, 66, 95-107 (2011)

Ayako KANAZAWA ${ }^{1,2}$, Chihiro MIYASITA ${ }^{2}$, Emiko OKADA ${ }^{2}$, Sumitaka KOBAYASHI ${ }^{2}$, Noriaki WASHINO ${ }^{2}$, Motoyuki YUASA ${ }^{2}$, Seiko SASAKI ${ }^{2}$, Eiji YOSHIOKA ${ }^{2}$, Futoshi MIZUTANI ${ }^{3}$,

Youichi CHISAKI ${ }^{3}$ and Reiko KISHI ${ }^{4}$

${ }^{1}$ Women's Junior College, Asahikawa University

${ }^{2}$ Department of Public Health Sciences, Graduate School

of Medicine, Hokkaido University

${ }^{3}$ IDEA Consultants, Inc.

${ }^{4}$ Center for Environmental and Health Sciences, Hokkaido University

Objectives: This study was performed to evaluate the levels of exposure to persistent organochlorine pesticides in pregnant women in Hokkaido.

Methods: Whole-blood samples were obtained from 70 pregnant women aged 17-39 years in Hokkaido and analyzed to quantify 29 organochlorine pesticides by gas chromatography/negative ion chemical ionization mass spectrometry and gas chromatography/high-resolution mass spectrometry. 
Results: Among 29 target compounds, 20 were detected in the wholeblood samples. Mirex, Parlar-26, and Parlar-50, which have never been used in Japan, were identified in all samples, as well as 11 compounds that have been used in Japan. Log-transformed concentrations of compounds with detection rates above $60 \%$ linearly correlated with each other $(p<0.01) . p, p^{\prime}$-DDE exhibited the highest concentration, with a geometric mean of $730 \mathrm{pg} / \mathrm{g}$ wet weight. From the results of the Jonckheere-Terpstra trend test, body weight or age was positively associated with the concentrations of several compounds.

Conclusions: We detected 22 organochlorine pesticides including pesticides with no history of use in Japan in the whole-blood samples from pregnant women in Hokkaido. Through long-distance transport mechanisms, these pollutants may distribute widely, and further surveillance of human blood, in addition to foods and the environment, should be conducted.

\section{Comparison of Long-Chain Polyunsaturated Fatty Acids in Plasma and Erythrocyte Phospholipids for Biological Monitoring}

Nippon Eiseigaku Zasshi, 66, 108-114 (2011)

Terue KAWABATA ${ }^{1}$, Kunihiko NAKAI ${ }^{2}$, Chie HAGIWARA ${ }^{1}$, Naoyuki KUROKAWA ${ }^{2}$, Katsuyuki MURATA ${ }^{3}$,

Kozue YAGINUMA ${ }^{2,4}$ and Hiroshi SATOH ${ }^{2}$

${ }^{1}$ Faculty of Nutrition, Kagawa Nutrition University

${ }^{2}$ Department of Environmental Health Sciences, Tohoku University Graduate School of Medicine

${ }^{3}$ Department of Environmental Health Sciences, Akita University Graduate School of Medicine

${ }^{4}$ Research Fellow of the Japan Society for the Promotion of Science

Objectives: Previous data have indicated that the erythrocyte membrane may be the preferred sample type for assessing long-chain polyunsaturated fatty acid (LCPUFA) contents in cardiac and cerebral membranes. In this epidemiological study, we examined whether plasma phospholipids can be used for accurate biological monitoring of the LCPUFA state or whether analysis of erythrocyte membrane phospholipids is indispensable.

Methods: (1) The analysis of LCPUFA contents in erythrocyte membrane phospholipids was conducted at baseline and after 1 and 3 days at $4^{\circ} \mathrm{C}$, and 21 days at $-40^{\circ} \mathrm{C}$, after blood drawing, and the changes in LCPUFA content were examined. (2) The LCPUFA compositions of plasma and erythrocyte phospholipids in 133 young women (18-30 years old) were examined and the relationships between the sample type and the levels of LCPUFAs were determined.

Results: Eicosapentaenoic acid (EPA), docosahexaenoic acid (DHA) and DHA/arachidonic acid (AA) and (EPA + DHA)/AA ratios in erythrocyte membrane phospholipids after 21 days of blood drawing significantly decreased compared with the corresponding baseline data. Regarding AA, EPA and DHA, a significant positive correlation was shown between levels of erythrocyte membrane phospholipids and plasma phospholipids (AA, $r=0.364$; EPA, $r=0.709$; DHA, $r=0.653$ ). The predictive value of plasma phospholipids for determining the highest concentration quartile in erythrocyte phospholipids was better in EPA $(70 \%)$ than in DHA $(55 \%)$ and AA $(42 \%)$.

Conclusions: The measurement of LCPUFA content in erythrocyte membrane phospholipids is necessary for accurate biological monitoring. We also found that LCPUFA in erythrocyte membrane phospholipids is stable in cold storage $\left(4^{\circ} \mathrm{C}\right)$ for 3 days after blood drawing.

\section{Bacterial Contamination of Mobile Phones Shared in Hospital Wards and the Consciousness and Behavior of Nurses about Biological Cleanliness}

Nippon Eiseigaku Zasshi, 66, 115-121 (2011)

Ikuharu MORIOKA ${ }^{1}$, Yuna TABUCHI ${ }^{1}$, Yuko TAKAHASHI ${ }^{1}$, Yuriko ODA $^{1}$, Masami NAKAI ${ }^{1}$, Aki YANASE ${ }^{2}$

and Chiyoko WATAZU ${ }^{3}$

${ }^{1}$ School of Health and Nursing Science, Wakayama Medical

University

${ }^{2}$ Wakayama Medical University Hospital

${ }^{3}$ Minami Wakayama Medical Center

Objectives: The purpose of this study was to clarify the contamination of mobile phones shared in hospital wards and its relationship with the consciousness and behavior of nurses about biological cleanliness. Methods: Samples from mobile phones were cultured to detect viable bacteria $(n=110)$ and Staphylococcus aureus $(n=54)$. A questionnaire survey was conducted on 110 nurses carrying mobile phones on the day of sampling.

Results: Viable bacteria were detected on $79.1 \%$ of the mobile phones, whereas $S$. aureus was detected on $68.6 \%$. All the nurses were aware of hand washing with water or alcohol after regular work, but $33.6 \%$ of the nurses were not conscious of hand washing with water or alcohol after using a mobile phone. There was a significant positive relationship between the frequency of using mobile phones and the number of hand washings with water or alcohol. A significant negative relationship was found between the detection of viable bacteria and the number of hand washings with alcohol. The results of logistic regression analysis showed that the detection of viable bacteria was related significantly with the number of hand washings with alcohol (Odds ratio, 0.350; 95\%CI, 0.143-0.857) and that the detection of $S$. aureus was related significantly with the frequency of using mobile phones (Odds ratio, 0.183; 95\% CI, 0.036-0.933).

Conclusions: It is important to be conscious of the fact that mobile phones shared in hospital wards are easily contaminated. Because hand washing with water or alcohol prevents the contamination of the mobile phones, nurses should take standard precautions after using mobile phones.

\section{Association of Indoor Air Quality with Physical Health of Users in a Newly Built School Building in a University}

Nippon Eiseigaku Zasshi, 66, 122-128 (2011)

Mihoko MORI $^{1}$, Kunio HARA ${ }^{2}$, Takashi MIYAKITA ${ }^{3}$

and Tatsuya ISHITAKE ${ }^{1}$

${ }^{1}$ Department of Environmental Medicine, Kurume University School of Medicine

${ }^{2}$ Faculty of Community Health Care, Teikyo Heisei University

${ }^{3}$ Faculty of Social Welfare, Kumamoto Gakuen University

Several users of a newly built school building in a university (new building) complained about their deteriorating physical health. We measured the air quality in the new building, an old school building and atmosphere in September 2007 and February 2008. We also conducted a questionnaire survey of subjective symptoms of users in the new building in February 2008. The university administrator took some remedial actions to improve the indoor air quality after the first measurement. In September 2007, the concentrations of total volatile organic compounds (TVOCs) and 2-ethyl-1-hexanol in the new building were higher than those in the old building and atmosphere. 
Moreover, the concentrations of TVOCs exceeded the Japanese recommended guideline values. In February 2008, the concentrations of these substances in the new building were lower than the previous values. Out of the 177 users who were surveyed regarding subjective symptoms, 59 responded to the survey. In September 2007, 21 users felt that their physical health had deteriorated, while in February 2008, 12 users felt no deterioration. However, nine users still complained about the deterioration of their physical health. It was suggested that the improvement in the indoor air quality may be influenced by the decrease in room temperature. Even if the concentrations of VOCs are below the recommended guideline values, users with an enhanced sensitivity towards VOCs may lose their tolerance to low-level VOCs. Our findings suggested that a survey of the changes in the subjective symptoms of users should be conducted to evaluate the improvement in the indoor air quality of newly built buildings. 